\title{
Are US Output Expectations Unbiased? A Cointegrated VAR Analysis in Real Time
}

\author{
by \\ Dimitrios Papaikonomou ${ }^{a}$ and Jacinta Pires ${ }^{b, *}$ \\ ${ }^{a}$ Ministry of Finance, Greece \\ ${ }^{b}$ Christ Church, University of Oxford, UK
}

\begin{abstract}
Real-time output and direct measures of expectations at different time horizons are analysed within a cointegrated VAR. We generally find expectations to be unbiased in the long-run with stationary expectational errors that persist between 4 and 14 quarters.
\end{abstract}

JEL Classifications: C15, C32, D84.

Keywords: Expectations, Survey Data, Real-Time Data, Long Run Structural VAR, Bootstrap Methods.

${ }^{*}$ We are most grateful to Kevin Lee and David Fielding for their invaluable help and guidance. Financial support from the portuguese institution "Fundacao para A Ciencia e Tecnologia", is also gratefully acknowledged. 


\section{Introduction}

Direct measures of expectations provide the means by which the expectations formation process can be explicitly studied. However, in most applications the time-series properties of the data have either been ignored or have not been explicitly accounted for. In particular, most tests of the Rational Expectations Hypothesis (REH) have been carried out under the implicit assumptions of stationarity of the expectational errors and long-run unbiasedness. ${ }^{1}$ Furthermore, rationality tests on output expectations, for instance, are frequently carried out using the most recently revised data on actual output, which can be quite dissimilar to the series available at the time the expectations were formed. ${ }^{2}$

This paper addresses both these issues in an analysis of the expectations formation process of US output. We account for the I(1) properties of the series by utilizing the multivariate cointegration framework of Johansen (1988) and its subsequent generalization in Pesaran, Shin and Smith (2000), in order to simultaneously model US actual output and direct measures of expected output over the period 1969q2-2001q2. Actual output is measured in real time, thus avoiding the criticisms associated with data revisions. Our direct measures of output expectations consist of the current-period, the one, two and threeperiod-ahead forecasts provided by the Survey of Professional Forecasters. We revisit the issues of (i) stationarity of the expectational errors, (ii) long-run unbiasedness and further investigate (iii) the short-run dynamics of output expectations and (iv) gain an insight as to how long the long run actually is.

\section{Modelling Framework}

\subsection{Definition of the Expectational Errors}

We consider the expectational errors $\eta_{i, t}, i=1,2,3,4, t=1,2, \ldots, T$, given by

$$
\begin{aligned}
& \eta_{1, t}={ }_{t} y_{t}^{e}-_{t+1} y_{t}, \eta_{2, t}={ }_{t} y_{t+1}^{e}-{ }_{t+2} y_{t+1}, \eta_{3, t}={ }_{t} y_{t+2}^{e}-{ }_{t+3} y_{t+2} \\
& \eta_{4, t}={ }_{t} y_{t+3}^{e}-_{t+4} y_{t+3}, t=1,2, \ldots, T
\end{aligned}
$$

where ${ }_{s} y_{p}$ denotes actual output at time $p$ available at time $s$ and the superscript " $e "$ denotes expectation, so that ${ }_{s} y_{p}^{e}$ is the expectation formed at time $s$ on the value of output

\footnotetext{
${ }^{1}$ One exception is Lahiri and Chun (1989).

${ }^{2}$ Patterson and Heravi (1991), for example, illustrate that different vintages of UK GDP components need not even be cointegrated.
} 
in time $p .^{3}$ The expectational errors in (1) may alternatively be written as

$$
\boldsymbol{\eta}_{t}=\boldsymbol{\beta}_{0}^{\prime} \mathbf{z}_{t}-\mathbf{A}(L) \Delta \mathbf{z}_{t} \quad, t=1,2, \ldots, T
$$

where $\boldsymbol{\eta}_{t}=\left[\eta_{1, t}, \eta_{2, t}, \eta_{3, t}, \eta_{4, t}\right]^{\prime}, \mathbf{z}_{t}=\left[{ }_{t} y_{t-1},{ }_{t} y_{t}^{e},{ }_{t} y_{t+1}^{e},{ }_{t} y_{t+2}^{e},{ }_{t} y_{t+3}^{e}\right]^{\prime}, \mathbf{A}(L)=A_{1} L^{-1}+$ $A_{2} L^{-2}+A_{3} L^{-3}+A_{4} L^{-4}, L$ being the lag-operator,

$$
\begin{aligned}
& A_{1}=\left[\begin{array}{lllll}
1 & 0 & 0 & 0 & 0 \\
1 & 0 & 0 & 0 & 0 \\
1 & 0 & 0 & 0 & 0 \\
1 & 0 & 0 & 0 & 0
\end{array}\right], A_{2}=\left[\begin{array}{lllll}
0 & 0 & 0 & 0 & 0 \\
1 & 0 & 0 & 0 & 0 \\
1 & 0 & 0 & 0 & 0 \\
1 & 0 & 0 & 0 & 0
\end{array}\right], A_{3}=\left[\begin{array}{llllll}
0 & 0 & 0 & 0 & 0 \\
0 & 0 & 0 & 0 & 0 \\
1 & 0 & 0 & 0 & 0 \\
1 & 0 & 0 & 0 & 0
\end{array}\right], \\
& A_{4}=\left[\begin{array}{lllll}
0 & 0 & 0 & 0 & 0 \\
0 & 0 & 0 & 0 & 0 \\
0 & 0 & 0 & 0 & 0 \\
1 & 0 & 0 & 0 & 0
\end{array}\right], \text { and } \boldsymbol{\beta}_{0}^{\prime}=\left[\begin{array}{lllll}
-1 & 1 & 0 & 0 & 0 \\
-1 & 0 & 1 & 0 & 0 \\
-1 & 0 & 0 & 1 & 0 \\
-1 & 0 & 0 & 0 & 1
\end{array}\right] .
\end{aligned}
$$

\subsection{Expectational Errors in a Cointegrating VAR Framework}

Provided that $\mathbf{z}_{t}=\left[{ }_{t} y_{t-1, t} y_{t}^{e},{ }_{t} y_{t+1}^{e},{ }_{t} y_{t+2}^{e},{ }_{t} y_{t+3}^{e}\right]^{\prime}$ is an $I(1)$ process with linear deterministic trending behaviour, it can be approximated by the following $\mathrm{VECM}^{4}$

$$
\Delta \mathbf{z}_{t}=\mathbf{a}_{0}+\mathbf{a}_{1} t+\Pi \mathbf{z}_{t-1}+\sum_{i=1}^{p-1} \Gamma_{i} \Delta \mathbf{z}_{t-i}+\mathbf{e}_{t}, \quad t=1,2, \ldots, T
$$

where

$$
\begin{gathered}
\mathbf{a}_{0}=-\Pi \boldsymbol{\mu}+[\Pi+\boldsymbol{\Gamma}(1)] \boldsymbol{\gamma}, \\
\mathbf{a}_{1}=-\Pi \boldsymbol{\gamma},
\end{gathered}
$$

$\mathbf{e}_{t} \sim \operatorname{IN}(\mathbf{0}, \Omega), \Omega$ positive-definite, $\boldsymbol{\mu}$ and $\boldsymbol{\gamma}$ are $5 \times 1$ vectors of unknown coefficients, $\boldsymbol{\Gamma}(1)=\mathbf{I}_{5}-\sum_{i=1}^{p-1} \Gamma_{i}, \Gamma_{i}, i=1, \ldots, p-1$ and $\Pi$ are $5 \times 5$ coefficient matrices and $0 \leq \operatorname{rank}[\Pi]=r<5$, so that $\Pi=\boldsymbol{\alpha} \boldsymbol{\beta}^{\prime}$ with $\boldsymbol{\alpha}, \boldsymbol{\beta}$ being $5 \times r$, full column rank.

The frequent assumption of stationarity of the expectational errors $\boldsymbol{\eta}_{t}$ defined in (2), requires that $\boldsymbol{\beta}=\boldsymbol{\beta}_{0}$. Under this hypothesis the expected value of $\boldsymbol{\eta}_{t}$ takes the form of

$$
E\left[\boldsymbol{\eta}_{t}\right]=\boldsymbol{\beta}_{0}^{\prime} \boldsymbol{\mu}+\left(\boldsymbol{\beta}_{0}^{\prime} \boldsymbol{\gamma}\right) t-\mathbf{A}(1) \boldsymbol{\gamma} \quad, t=1,2, \ldots, T
$$

${ }^{3}$ Actual output is GNP up to 1991q4 and GDP thereafter, following a change in the survey's questionnaire. We use the 15-day vintage as it reflects more accurately the information available to the respondents of the survey when the questionnaires are sent out. All variables are in natural logarithms.

${ }^{4}$ See for example Pesaran, Shin and Smith (2000). 
where $\mathbf{A}(1)=\sum_{i=1}^{4} A_{i}$ and $A_{i}, i=1,2,3,4$ were defined in (3). Unbiasedness requires $E\left[\boldsymbol{\eta}_{t}\right]=\mathbf{0}$, which in the current framework corresponds to $\boldsymbol{\beta}_{0}^{\prime} \boldsymbol{\gamma}=\mathbf{0}$, also known as the cotrending hypothesis, and $\mathbf{A}(1) \boldsymbol{\gamma}=\boldsymbol{\beta}_{0}^{\prime} \boldsymbol{\mu}$. Since the VECM in (4) does not provide estimates of $\boldsymbol{\gamma}$ and $\boldsymbol{\mu}$, the restrictions $\mathbf{A}(1) \boldsymbol{\gamma}=\boldsymbol{\beta}_{0}^{\prime} \boldsymbol{\mu}$ cannot be tested in general. However, provided that $\boldsymbol{\beta}=\boldsymbol{\beta}_{0}$ and for $\boldsymbol{\gamma}=\left[\gamma_{1}, \gamma_{2}, \gamma_{3}, \gamma_{4}, \gamma_{5}\right]^{\prime}$, co-trending is equivalent to $\boldsymbol{\gamma}=\left[\gamma_{1}, \gamma_{1}, \gamma_{1}\right.$, $\left.\gamma_{1}, \gamma_{1}\right]^{\prime}$, where $\gamma_{1}$ is the mean of $\Delta_{t} y_{t-1}$. In other words, the variables in $\mathbf{z}_{t}$ are not only driven by a common stochastic trend, but they also share the same deterministic trend with ${ }_{t} y_{t-1}$. Therefore, given $\boldsymbol{\beta}=\boldsymbol{\beta}_{0}, \boldsymbol{\beta}_{0}^{\prime} \boldsymbol{\gamma}=\mathbf{0}$ and an estimate of $\hat{\gamma}_{1}$, long-run unbiasedness imposes through (5) the following set of testable restrictions

$$
\mathbf{A}(1) \hat{\gamma}=\left(\boldsymbol{\alpha}^{\prime} \boldsymbol{\alpha}\right)^{-1} \boldsymbol{\alpha}^{\prime}\left[\boldsymbol{\Gamma}(1) \hat{\gamma}-\mathbf{a}_{0}\right]
$$

\section{Empirical Analysis}

Our data on $\left.\mathbf{z}_{t}={ }_{t} y_{t-1, t} y_{t, t}^{e} y_{t+1, t}^{e} y_{t+2}^{e},{ }_{t} y_{t+3}^{e}\right]^{\prime}$ are quarterly and cover the period 1968q42001q2. The empirical analysis is based on the VECM given by (4), augmented by the dummy vector $\mathbf{D}_{t}=\left[d 71 q 4_{t}, d 74 q 4_{t}, d 81 q 1_{t}, d 96 q 1_{t}, d 99 q 4_{t}\right]^{\prime}$, where $d 71 q 4_{t}$ takes the value of one in 1971q4 and zero otherwise and the remaining variables are similarly defined. The dummies $d 71 q 4_{t}$ and $d 74 q 4_{t}$ are intended to capture the slowdown in economic activity at the end of 1971 and 1974, while $d 81 q 1_{t}, d 96 q 1_{t}$ and $d 99 q 4_{t}$ are controlling for the effects of the comprehensive revisions in GDP that took place in December 1980, December 1995 and August 1999. The employment of multivariate ADF tests and the $L R$ statistics for testing the restriction of the trend coefficients according to (6) (available on request) indicated that, at conventional significance levels the vector $\mathbf{z}_{t}$ can indeed be described as an $I(1)$ process with linear deterministic trending behaviour, irrespective of the choice of $r$. The lag-length, $p$, was set equal to 2 after testing for significance of additional lags within an unrestricted $V A R(8)$ in the level of $\mathbf{z}_{t}$, as well as with the use of the $A I C$ and $S B C$.

\subsection{Properties of the Expectational Errors}

Table 1 reports the $\lambda$-trace and maximal eigenvalue cointegration rank statistics. In general, the simulated critical values verify the presence of a finite-sample bias in favour of rejection reported, for example, by Harris and Judge (1998). Nevertheless, this appears to make no qualitative difference to the outcome of the tests, as all the evidence appear to be clearly in favour of our economic priors that $r=4$.

Table 2 reports the tests of three sets of over-identifying restrictions on $\boldsymbol{\beta}_{*}^{\prime}=\left[\boldsymbol{\beta}^{\prime},-\boldsymbol{\beta}^{\prime} \boldsymbol{\gamma}\right]$. The first set, denoted $R_{O V 1}$, corresponds to the test of $\boldsymbol{\beta}=\boldsymbol{\beta}_{0}$, which cannot be rejected even asymptotically. The set of restrictions denoted as $R_{O V 3}$ corresponds to the joint hypothesis $\boldsymbol{\beta}=\boldsymbol{\beta}_{0}$ and $\boldsymbol{\beta}_{0}^{\prime} \boldsymbol{\gamma}=\mathbf{0}$. Asymptotically this joint hypothesis is clearly rejected. 
With reference to the simulated finite-sample distribution, however, rejection is avoided at the $5 \%$ level, although not at the $10 \%$ level. ${ }^{5}$ Stronger evidence is obtained for the hypothesis that the trend coefficients are absent from the cointegrating relations corresponding to $\eta_{1, t}$, $\eta_{2, t}$ and $\eta_{3, t}$ alone, denoted as $R_{O V 2}$. These results indicate that the joint hypothesis $\boldsymbol{\beta}=\boldsymbol{\beta}_{0}$ and $\boldsymbol{\beta}_{0}^{\prime} \boldsymbol{\gamma}=\mathbf{0}$ appears to be consistent with the data as far as $\eta_{1, t}, \eta_{2, t}$ and $\eta_{3, t}$ are concerned. Regarding $\eta_{4, t}$ the evidence appear to be less conclusive and raise the suspicion that it could possibly follow a near trend-stationary process. With reference to expression (7), such a property would imply a particularly disappointing performance on the part of economic agents in predicting the tree-period-ahead level of output, as the mean forecast error would be a linear function of time. In the light of this and considering the evidence reported in, inter alia, Johansen (2000) regarding the finite-sample bias of $\chi^{2}$ tests, we have decided to put more weight on the simulation results and maintain the co-trending hypothesis.

The diagnostic and descriptive statistics of the VECM estimated subject to $\boldsymbol{\beta}=\boldsymbol{\beta}_{0}$ and $\boldsymbol{\beta}_{0}^{\prime} \gamma=\mathbf{0}$ (available on request) revealed no signs of model misspecification at conventional levels of significance. The restrictions in (8) were tested for $\hat{\gamma}_{1}=0.0075176$ and the relevant Wald statistic was found to be 2.691 with an asymptotic $5 \%$ critical value of 9.49 , thus verifying that expectations are indeed unbiased. However, it should be stressed that this result rests on the joint assumption $\boldsymbol{\beta}=\boldsymbol{\beta}_{0}$ and $\boldsymbol{\beta}_{0}^{\prime} \boldsymbol{\gamma}=\mathbf{0}$, for which the evidence were less convincing as far as $\eta_{4, t}$ is concerned.

\subsection{Dynamic Behaviour of Output Expectations and Expectational Er- rors}

Figure 1 plots the Generalized Impulse Responses (GIR) for $\mathbf{z}_{t}$ to a one-standard error innovation in ${ }_{t} y_{t-1}$, which in this case are exactly equivalent to the more standard Orthogonalized Impulse Responses (OIR) ${ }^{6}$ The impact effect of the shock is to raise real-time output by $0.62 \%$ and output expectations between $0.668 \%$ and $0.697 \%$. After approximately 14 quarters all variables stabilize at $0.756 \%$ higher than their pre-shock level. This illustrates the $I(1)$ properties of the variables that cause a given shock to have a permanent effect, as well as the stationary nature of the expectational errors that eventually eliminates the gap between actual and expected output.

The response of the expectational errors $\boldsymbol{\eta}_{t}$ is explicitly illustrated in Figure 2. On impact, the rise in ${ }_{t} y_{t-1}$ results in underprediction which is greater the longer the forecast horizon and ranges between $-0.10 \%$ and $-0.16 \%$. The expectational errors persist between 4 and 14 quarters, with the adjustment process being faster the shorter the forecast horizon.

\footnotetext{
${ }^{5}$ Non-parametric simulation is based on Fachin (2000).

${ }^{6}$ For a proof of the fact that GIR $=$ OIR when considering a shock in the first variable of the system see Pesaran and Shin (1998).
} 


\section{Concluding Remarks}

Our findings have confirmed the quite frequent assumption of stationary expectational errors. Furthermore, all expectations may reasonably be argued to be unbiased in the long run, although the evidence is weaker in the case of the three-period ahead forecast. Expectational errors are found to persist between 4 and 14 quarters, with the adjustment process being faster the shorter the forecast horizon. 


\section{References}

Fachin, S., 2000, Bootstrap and Asymptotic Tests of Long-Run Relationships in Cointegrated Systems, Oxford Bulletin of Economics and Statistics, 62, 4, 543-551.

Harris, R.I.D. and G. Judge, 1998, Small Sample Testing for Cointegration using the Bootstrap Approach, Economics Letters, 58, 31-37.

Johansen, S., 1988, Statistical Analysis of Cointegration Vectors, Journal of Economic Dynamics and Control, 12, 231-254.

Johansen, S. , 2000, A Bartlett Correction Factor for Tests on the Cointegrating Relations, Econometric Theory 740-778.

Lahiri, K. and T.S. Chun, 1989, Some Tests for Unbiasedness in the Long-Run Using Survey Data, International Economic Journal, 3, 27-42.

Patterson, K.D. and S.M. Heravi, 1991, Are Different Vintages of Data on the Components of GDP Co-integrated? Some Evidence for the United Kingdom, Economics Letters, 35, 409-413.

Pesaran, M.H. and Y. Shin, 1998, Generalised Impulse Response Analysis in Linear Multivariate Models, Economics Letters, 58, 17-29.

Pesaran, M.H., Y. Shin and R.J. Smith , 2000, Structural Analysis of Vector Error Correction Models with Exogenous I(1) Variables, Journal of Econometrics, 97, 293343. 
Table 1 - Cointegration Rank Statistics

\begin{tabular}{|c|c|c|c|c|c|}
\hline \multirow[b]{2}{*}{$H_{0}$} & \multirow[b]{2}{*}{$H_{1}$} & \multicolumn{2}{|c|}{$\lambda$-Trace } & \multicolumn{2}{|c|}{ Maximum Eigenvalu } \\
\hline & & Statistic & $5 \% \mathrm{cv}$ & Statistic & $5 \% \mathrm{cv}$ \\
\hline$r=0$ & $r=1$ & 223.03 & $\begin{array}{l}87.17 \mathrm{a} \\
87.48 \mathrm{p} \\
91.77 \mathrm{n} \\
\end{array}$ & 80.88 & $\begin{array}{l}37.86 \mathrm{a} \\
40.98 \mathrm{p} \\
41.12 \mathrm{n} \\
\end{array}$ \\
\hline$r \leq 1$ & $r=2$ & 142.15 & $\begin{array}{l}63.00 \mathrm{a} \\
60.69 \mathrm{p} \\
66.44 \mathrm{n} \\
\end{array}$ & 65.74 & $\begin{array}{l}31.79 \mathrm{a} \\
33.55 \mathrm{p} \\
34.56 \mathrm{n} \\
\end{array}$ \\
\hline$r \leq 2$ & $r=3$ & 76.41 & $\begin{array}{l}42.34 \mathrm{a} \\
44.15 \mathrm{p} \\
44.21 \mathrm{n}\end{array}$ & 43.93 & $\begin{array}{l}25.42 \mathrm{a} \\
27.65 \mathrm{p} \\
27.74 \mathrm{n}\end{array}$ \\
\hline$r \leq 3$ & $r=4$ & 32.48 & $\begin{array}{l}25.77 \mathrm{a} \\
24.72 \mathrm{p} \\
25.90 \mathrm{n}\end{array}$ & 24.23 & $\begin{array}{l}19.22 \mathrm{a} \\
19.37 \mathrm{p} \\
20.01 \mathrm{n}\end{array}$ \\
\hline$r \leq 4$ & $r=5$ & 8.24 & $\begin{array}{l}12.39 \mathrm{a} \\
12.78 \mathrm{p} \\
12.52 \mathrm{n} \\
\end{array}$ & 8.24 & $\begin{array}{l}12.39 \mathrm{a} \\
12.78 \mathrm{p} \\
12.52 \mathrm{n} \\
\end{array}$ \\
\hline
\end{tabular}

Notes: Small-sample results are based on a bootstrap with 20,000 simulations. "p" and "n" indicate parametric and non parametric respectively, while " $\mathrm{a}$ " indicates asymptotic critical value.

Table 2 - Tests of Restrictions on the Cointegra ting Parameters

\begin{tabular}{|c|c|c|c|c|c|}
\hline Restrictions & LR-statistic & \multicolumn{2}{|c|}{ Asymptotic } & \multicolumn{2}{c|}{ Small Sample } \\
\hline \multirow{3}{*}{$R_{O V 1}$} & \multirow{3}{*}{5.28} & $5 \% \mathrm{cv}$ & $10 \% \mathrm{cv}$ & $5 \% \mathrm{cv}$ & $10 \% \mathrm{cv}$ \\
\cline { 3 - 6 }$R_{O V 2}$ & 9.78 & 9.49 & 7.78 & - & - \\
\cline { 3 - 6 }$R_{O V 3}$ & 19.08 & 14.07 & 12.02 & - & - \\
\cline { 3 - 6 } & & 15.51 & 13.36 & $\begin{array}{l}21.17 \mathrm{p} \\
21.39 \mathrm{n}\end{array}$ & $\begin{array}{c}18.29 \mathrm{p} \\
18.36 \mathrm{n}\end{array}$ \\
\hline
\end{tabular}

Notes: $R_{\text {OVi }}, i=1,2,3$, are defined in the text. Small -sample results are based on a bootstrap with 10,000 simulations. "p" and "n" indicate parametric and non-parametric respectively.
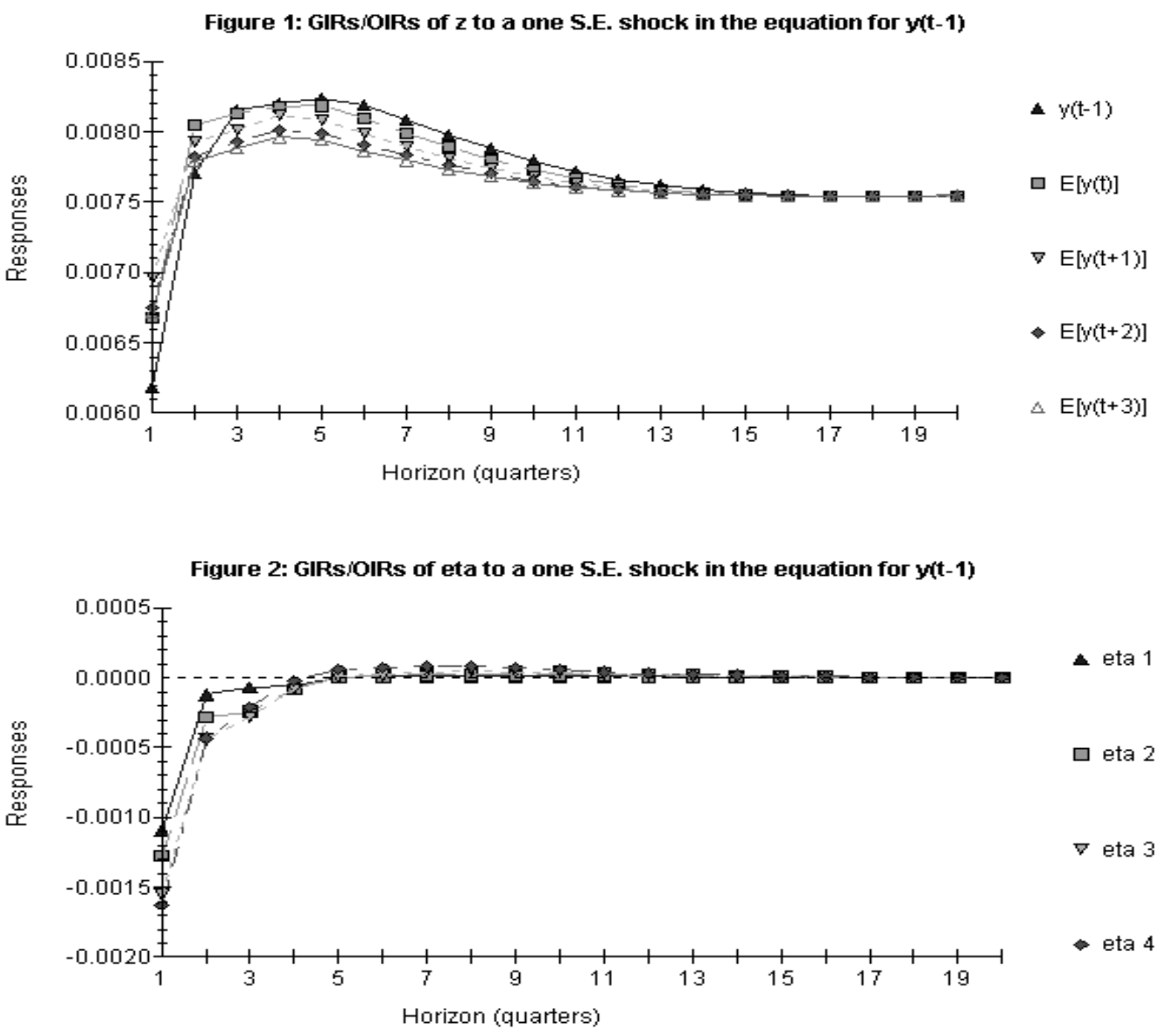